\title{
Secuenciación de Nueva Generación: ¿Es Factible su Implementación en el Ámbito Forense Colombiano?
}

Next-Generation Sequencing: Is Its Implementation Feasible in the Colombian Forensic Field?

Diego Luis Gaviria Vélez¹, Juan Alejandro Calderón Hernández², Aura María Gil-Villa

Autor correspondencia: Diego Luis Gaviria Vélez. Correo electrónico: dgvelez@gmail.com

Tipo de artículo: Artículo de revisión Recibido: 10 de octubre de 2020. Aprobado: 16 de noviembre de 2021.

DOI: https://doi.org/10.53995/25390147.1012

Resumen: Esta revisión realiza una aproximación metodológica cercana a los retos que enfrentan las instituciones de nuestro país involucradas en el estudio genético-forense, relacionado con el impacto de las nuevas tecnologías en lo referente al análisis de los marcadores genéticos empleados para la identificación de tipo forense, como filiaciones o uni-procedencias, utilizadas comúnmente por los laboratorios del país. En la actualidad, se utilizan sistemas de identificación por medio de ADN de tipo microsatélite (STR), que aportan máximo 45 marcadores autosómicos y sexuales, que entregan información suficiente para realizar una identificación veraz de un individuo; no obstante, se presentan situaciones en que los resultados esperados no son los óptimos, debido a la degradación que pueden presentar las muestras, contaminaciones y bajo contenido de ADN. Hoy por hoy, se trabaja en una modificación al empleo y manejo de estos marcadores para dar mejor rendimiento en la obtención de perfiles genéticos en todo tipo de muestras, generando resultados basados en secuencias específicas de nucleótidos, mejorando los perfiles genéticos a estudiar, ya que se amplía el número de marcadores genéticos, se reduce el tiempo de análisis y la fiabilidad de los resultados obtenidos para realizar los respectivos cotejos de interés forense.

Palabras clave: Secuenciación de nueva generación, ADN, STR, perfiles genéticos, ciencias forenses, Colombia.

Como citar este artículo: Gaviria Vélez, D., Calderón Hernández, J., y Gil-Villa, A. (2021). Secuenciación de Nueva Generación: ¿Es Factible su Implementación en el Ámbito Forense Colombiano?. Memorias Forenses, 5, 75-91. DOl: https://doi.org/10.53995/25390147.1012.

\footnotetext{
1 Biólogo. Especialista y Magíster en Ciencias Forenses y Criminalística. Facultad de Derecho y Ciencias Forenses. Tecnológico de AntioquiaInstitución Universitaria. Medellín-Colombia. Coordinador de la unidad de secuenciación Gentech Biosciences. Medellín, Antioquia. Correo electrónico: dgvelez@gmail.com

2 Estudiante de Profesional en Criminalística. Facultad de Derecho y Ciencias Forenses. Grupo de Investigación BISMA. Tecnológico de Antioquia-Institución Universitaria. Medellín-Colombia. Correo electrónico: Juan.calderon69@correo.tdea.edu.co

3 Docente investigadora. Facultad de Derecho y Ciencias Forenses. Grupo de Investigación BISMA. Tecnológico de Antioquia-Institución Universitaria. Medellín-Colombia. Correo electrónico: aura.gil@tdea.edu.co
} 


\title{
Next-Generation Sequencing: Is Its Implementation Feasible in the Colombian Forensic Field?
}

\begin{abstract}
This review, makes an method approximate sketch of the challenges facing the institutions of our country involved in the genetic-forensic study related to the impact of new technologies in relation to the analysis of the genetic markers used for the identification of forensic type used as filiations or uni-provenances, commonly used by forensic genetics laboratories in the country. Currently, identification systems are used by means of DNA of microsatellite type (STR), which provide a maximum of up to 45 autosomal and sexual genetic markers, which provide sufficient information to make a true identification of an individual; nevertheless, there are situations in which the expected results are not the most optimal, due to the degradation that the samples may present, contaminations and low DNA content. Currently, the use and management of genetic markers is changed to give a better performance in obtaining genetic profiles in all types of samples, generating results based on specific nucleotide sequences, and in this way improving the genetic profiles to be studied, and that the number of genetic markers is extended, the analysis time and the reliability of the results obtained to perform the respective genetic comparisons are reduced.
\end{abstract}

Key Word: Next-generation sequencing (NGS), DNA, STR, genetic data bases, forensic genetic, Colombia.

\section{Sequenciação de Nova Geração: É Possível a sua Implementação no Ambito Forense Colombiano?}

Resumo: Esta revisão apresenta uma aproximação metodologica aos desafíos com os quais lidan as instituições em nosso país as quais estão involvidas no estudo genético-forense, relacioado com o impatos das novas tecnologías no referente ao análise dos marcadores genéticos utilizados nas identificações do tipo forense, como afiliações ou uni-procedencias, as quais são utilizadas de forma comun nos laboratorios do país. Atualmente, são utilizados sistemas de identificçaõ do ADN tipo microsatélite (STR's), os quais aportan no máximo 45 marcadores autossômicos e sexuais que entregam suficiente informação para realizar uma identificaçao verdadeira de um individuo; no entanto, se apresentan situações nas quais os resultados esperados não são ótimos, devido á degradação que poden apresentar as amostras, contaminação e baixo conteúdo do ADN. Atualmente, são desenvolvidas modificações no uso e gestão destes marcadores para dar um melhor rendimento na obtenção dos perfis genéticos em todo tipo de amostras, gerando resultados baseados nas sequencias específicas dos nucleotídeos, melhorando os perfis genéticos á serem estudados, dado que o número de marcadores genéticos é estendido, e o tempo de análise e a cofiabilidade dos resultados obtidos para realizar as respetivas comparações é reducido.

Palavras chave: Sequenciação de nova geraçaõ, ADN, STR, perfis genéticos, ciência forense, Colombia. 
En genética forense, el uso de microsatélites o secuencias cortas repetidas en tándem (STR) ha sido, durante los últimos 20 años, la principal herramienta para la identificación humana; esta ha sido empleada en desastres masivos (Sudoyo et al., 2008), identificación de restos óseos (), criminalística), y en la determinación de relaciones heredo-biológicas entre individuos como maternidad, paternidad, entre otras (GarcíaAceves et al., 2018). Los anteriores procesos se han realizado convencionalmente, mediante la amplificación de STR por la técnica de reacción en cadena de la polimerasa (PCR), y posterior análisis a través de electroforesis capilar (Butler, 2015a).

Hasta la fecha, han sido numerosos y significativos los procesos de identificación humana que han tenido éxito, los cuales han mejorado con el tiempo, incorporando avances científicos (Afrifah et al., 2020). Importantes hechos históricos han usado la genética forense como herramienta fundamental para la identificación humana. Por su parte, el número de marcadores genéticos ha aumentado debido al nivel de certeza necesaria en un momento histórico específico; así, en el atentado terrorista cometido el 11 de septiembre del 2001, en la ciudad de New York-U.S., Los laboratorios del FBI aumentaron el número de marcadores STR a 16 en los procesos de identificación de las víctimas, precepto aceptado por la comunidad internacional en ese momento (Grupo Español y Portugués de la ISFG, s.f.).

Lo anterior, permitió la integración de protocolos de trabajo en los laboratorios de identificación genética en los Estados Unidos (Biesecker, 2005). De igual manera, casos en criminalística que han implicado homicidios o violencia sexual han utilizado técnicas de biología molecular en conjunto con análisis de STR, permitiendo su resolución, fortaleciendo y favoreciendo de manera exitosa los procesos judiciales (Tozzo et al., 2018). En actualidad, el número de marcadores disponibles para realizar este tipo de procedimientos se ha ampliado, hasta tener disponibles un aproximado de 58 marcadores genéticos de tipo autosómico y sexual, que han sido útiles y aceptados por la comunidad forense internacional (Katsanis \& Wagner, 2012; Butler, 2015a).
Con el auge de la tecnología de secuenciación de nueva generación o secuenciación masiva en paralelo- NGS, con la cual se pueden realizar secuencias completas de genomas, se han desarrollado sistemas de identificación con la capacidad de rastreo de hasta 230 marcadores genéticos, donde se incluyen marcadores de tipo STR autosómico y sexual, polimorfismos de un solo nucleótido-SNP's, que corresponden a polimorfismos de un único nucleótido; marcadores de ancestría, así como fenotípicos (Ibarra, 2015). Estos procesos se han convertido en una herramienta de importante utilidad en el área de la genética forense, ya que, al aumentar el número de marcadores, el estudio probabilístico es mucho más robusto, proporcionando resultados más veraces y confiables (Iwamura et al., 2016).

En Colombia, los encargados de realizar análisis mediante genética forense son los laboratorios del Instituto Nacional de Medicina Legal y Ciencias Forenses (INMLCYF), los laboratorios del Cuerpo Técnico de Investigaciones (CTI) de laFiscalía General de la Nación y, en menor medida, los laboratorios de genética de la Policía Nacional de Colombia, según lo dispuesto por la Ley 1408 de 2010, donde se genera la normatividad para el análisis de los restos humanos con fines de identificación en el conflicto armado, usando todas las herramientas previstas para su estudio. En estos laboratorios de genética forense del Instituto Nacional de Medicina Legal y Ciencias Forenses, el trabajo se divide dependiendo el origen y las características del material biológico a evaluar, ya sea componentes líquidos como sangre, saliva y semen o sólidos como pelos y huesos. Así, los grupos de criminalística se encargan del manejo genético de muestras provenientes de delitos como homicidios, delitos sexuales, entre otros, en donde se procesa todo tipo de muestras de origen humano; por su parte, los grupos de identificación de restos óseos se encargan de valorar los materiales provenientes de los delitos de desaparición forzada y eventos con fines diferentes a hechos delictivos (Villalobos Rangel, 2017).

Teniendo en cuenta, que en Colombia se presenta un número elevado de casos que involucran restos óseos de las víctimas del conflicto y la violencia en 
general, este tipo de análisis se realiza por medio de diferentes disciplinas como la antropología y la odontología. No obstante, también se ha hecho necesario su paso por un análisis de tipo genético para obtener mayor cantidad de información de las osamentas en estudio (Paredes y Romero, 2009; Instituto Nacional de Medicina Legal y Ciencias Forenses, 2020) y, de esta manera, poder realizar una determinación fehaciente de la identidad de los individuos y generar así, mayor certeza al momento de la entrega a los familiares de las víctimas (Penacino et al., 2003; Penacino, 2003). Las instituciones encargadas de realizar este tipo de valoraciones deben recurrir a la implementación de nuevas tecnologías en genética forense para el mejoramiento de procesos y efectividad de estos frente a los nuevos retos que se presenta en la identificación humana en nuestro país. Por lo anterior, el objetivo de la presente revisión de la literatura es describir métodos convencionales de secuenciación como el análisis de STR empleados en Colombia, el método de Secuenciación de Nueva Generación (NGS) y, de esta manera, determinar la utilidad y la conveniencia de la implementación de esta última metodología en el ámbito forense colombiano.

\section{Metodología}

Se realizó una búsqueda de información publicada en las bases de datos reconocidas por la comunidad científica como PubMed y Science Direct; se incluyó información de artículos publicados en los últimos 20 años. Se utilizaron los siguientes descriptores $\mathrm{MeSH}, \mathrm{DNA}, \mathrm{STR}, \mathrm{NSG}$, forensic genetic y genetic data bases. Adicionalmente, se usó los términos comerciales MiSeq y ForenSeq, y finalmente, se tuvieron en cuenta diferentes configuraciones de búsqueda, las cuales se detallan a continuación: "(forensic) OR (forensic genetics) AND (microsatellite repeats) OR repeat, (short tandem) AND (next sequencing generation)"; "(forensic) OR (forensic genetics) AND (microsatellite repeats) OR repeat, (short tandem) AND (next sequencing generation) OR (next sequencing generation) AND (reproducibility of results) OR (sensitivity and specificity) OR (accuracy) AND (clinical trial) OR (epidemiological studies)"; "(forensic) OR (forensic genetics) AND (microsatellite repeats) OR repeat, (short tandem) AND (next sequencing generation) OR (next sequencing generation) AND (reproducibility of results) OR (sensitivity and specificity) OR (accuracy) AND (clinical trial) OR (epidemiological studies)"; "(clinical trial) OR epidemiological studies"; "(reproducibility of results) OR (sensitivity and specificity) OR accuracy"; "(next sequencing generation) OR next sequencing generation"; "(microsatellite repeats) OR repeat, short tandem"; "(forensic) OR forensic genetics", "epidemiological studies"; "clinical trial"; "accuracy"; "sensitivity and specificity"; "search reproducibility of results"; "microsatellite repeats"; "repeat, short tandem"; "forensic genetics"; "forensic"; "next sequencing generation".

\section{Marcadores Genéticos como Herramientas de Identificación}

La identificación forense ha sido uno de los principales retos para la ciencia; en el siglo $X X$, se adaptaron nuevos descubrimientos en el área de la biología celular y la medicina a la criminalística a través del uso de marcadores genéticos heredables, como los grupos sanguíneos (Watkins, 1980) y las isoenzimas (Rees \& Rothwell, 1975); estos marcadores son lo suficientemente polimórficos y su distribución es estadísticamente significativa para ser usado como una herramienta de identificación forense, aunque no es lo suficientemente veraz para generar una identidad fehaciente, ya que es bajo su poder de discriminación (Kesseli et al., 1991). Por esta razón, se comenzó el estudio de métodos encaminados al uso de la biología molecular para mejorar la capacidad de individualización y perfilación de individuos. A continuación, se detallan los fundamentos conceptuales de algunas técnicas moleculares que son indispensables para la amplificación y análisis genético basado en STR y librerías NGS, y posteriormente, se evaluará la pertinencia de esta última para su implementación en el ámbito forense colombiano. 


\section{Técnicas de Biología Molecular Previas al Análisis por STR y NGS}

Extracción de ADN. Los procesos de extracción de ADN, varían según la necesidad del investigador, teniendo en cuenta el tipo de muestra, su posible degradación y contaminantes, lo que determina el tipo de extracción a emplear. Las técnicas más usadas para la extracción de ácidos nucleicos en el mundo son:

a) La técnica de fenol-cloroformo: en este procedimiento se aprovecha la inmiscibilidad que tienen ambos compuestos, generando una solución de dos fases: acuosa, en donde se encuentran los ácidos nucleicos; y una segunda que se precipita y que contendrá grasas, proteínas y otros inhibidores del proceso de amplificación por PCR. Este método es usado ampliamente en extracciones de diferentes tipos de tejidos, como huesos, músculos y demás tejidos biológicos de diferente origen (Alvis-Arango, 2016; Mccord, 2018).

b) Extracción de ácidos nucleicos por resinas quelantes: estas sirven como intercambiadoras de iones que funcionan realizando un atrapamiento de estos, pero metálicos polivalentes, los cuales actúan como catalizadores en la ruptura de ADN a altas temperaturas e inhibiendo la amplificación en la PCR. Tienen la capacidad de remover cualquier catión presente en una solución atrayéndolos y pegándose a la superficie de los gránulos de resina; principalmente, enlazan iones de magnesio, necesarios para que las nucleasas digieran el ADN, protegiendo así la integridad de la molécula hereditaria, y finalmente, por centrifugación se obtienen el ADN del sobrenadante (Brito et al., 2019; García García et al., 2008; de Armas et al., 2011). Los procedimientos manuales de extracción como las resinas quelantes o el fenolcloroformo y los tiempos de procesamiento de las muestras pueden variar entre 45 hasta 480 minutos, dependiendo la muestra. El costo promedio comercial para la ejecución de estos procedimientos de manera manual se encuentra entre 0,75 a 1,25 dólares para resinas quelantes y 0,5 a 2,0 dólares para la extracción fenol-cloroformo. Para su ejecución automatizada, sea por columnas de sílice, en donde se presentan perlas de sílice que se encuentran a $\mathrm{pH}$ ácido que favorece a los ácidos nucleicos para su preservación, el ADN se adhiere a las perlas por su afinidad y después de una serie de lavados se utiliza un imán para atraerlas. El tiempo de proceso puede variar entre 45 a 90 minutos por muestra, con un costo que oscila entre 15 y 45 dólares por cada una (Alejos et al., 2008; DNeasy blood \& tissue handbook, 2006).

Cuantificación de ADN. Sucede posterior al proceso de extracción, es necesaria para observar la calidad y cantidad de ácidos nucleicos presentes en la muestra, permitiendo determinar, antes de la amplificación del ADN y en términos de calidad, si el ADN ha sufrido procesos de degradación o presencia de inhibidores (Butler, 2015a). Este proceso se puede llevar a cabo por diferentes técnicas, como la PCR en tiempo real (qPCR), que permite monitorear de manera continua la producción de un amplificado durante una PCR; el método se basa en la detección y cuantificación de la fluorescencia de una molécula sonda con el paso del tiempo (Butler, 2015a). Esta técnica incluye la detección de zonas ligadas al cromosoma Y; la duración de este proceso se tarda entre 30 y 50 minutos, según el modelo del termociclador, con un costo que puede variar por muestra entre 8 hasta 12 dólares (QiaGen, 2017). Por su parte, la espectrofotometría UV-visible es una técnica analítica que permite determinar la concentración de un compuesto en solución, teniendo en cuenta que las moléculas absorben las radiaciones electromagnéticas y, a su vez, la cantidad de luz absorbida depende de forma lineal de su concentración (QiaGen, 2018; ThermoFisher Scientific, 2000). Para realizar este tipo de medidas, se emplea un espectrofotómetro, en el que se puede seleccionar la longitud de onda de la luz que pasa por una solución y medir la cantidad de luz absorbida por la misma; el ADN es analizado teniendo en cuenta una relación de longitud de onda 260-280 nm con respecto a una curva de calibración previamente establecida (Watson et al., 2007); esta técnica ha variado ostensiblemente con el tiempo hasta generar excelentes resultados y costos atractivos aproximados de 0,5 a 1,0 dólares, y el tiempo de análisis es de aproximadamente un segundo. 
Ahora bien, teniendo en cuenta que en el ámbito forense se requiere una serie de técnicas que buscan detectar la presencia de marcadores genéticos, es esencial la implementación de pasos posteriores de identificación como los detallados a continuación.

Amplificación de STR. La información genética contenida en los seres vivos se encuentra al interior de las células, dentro de la molécula del ADN; esta se presenta como una doble hebra conformado por nucleótidos (conocidos como adenina, timina, citosina y guanina), unidos químicamente entre sí hasta formar la doble hélice (Watson et al., 2007). El ADN se caracteriza por tener regiones codificantes y otras no codificantes para proteínas, en estas últimas zonas se encuentran regiones con secuencias altamente repetitivas, las cuales se heredan de acuerdo con los postulados de Mendel; según el número de repeticiones, son conocidas como marcadores de tipo minisatélites o VNTR's, donde se observan entre 6 a 100 pares de bases, y los marcadores de tipo microsatélites o STR, donde se presentan entre 3 a 5 repeticiones en tándem. Los marcadores genéticos tipo STR se encuentran localizados en casi la totalidad de los cromosomas humanos, la gran mayoría de estos son usados y reconocidos por la comunidad científica forense internacional (Katsanis \& Wagner, 2012; Butler, 2015b).

Una de las principales técnicas usadas en biología molecular e incorporada al análisis de STR en genética forense, es la PCR, desarrollada en los 80 's, la cual aprovecha dos características propias del ADN, el desenrollamiento de esta molécula por cambios térmicos y su renaturalización; así como la acción de la enzima ADN polimerasa en el proceso de replicación de la molécula, generando de esta manera, múltiples copias de una sección de ADN de interés (Watson et al., 2007). Este proceso inicia con componentes básicos como ADN polimerasa, dideoxinucleotidos e ion magnesio, cebadores (forward y reverse) y una muestra de ADN de interés. Al inicio, se requiere una temperatura elevada $\left(96^{\circ} \mathrm{C}\right)$, que provoca la desnaturalización del ADN de doble cadena; el siguiente paso $\left(35-60^{\circ} \mathrm{C}\right)$ ayudará a la alineación de los cebadores que consisten en una secuencia corta de ADN de cadena simple que se utiliza durante la PCR como iniciador de la reacción, en las zonas que presentan complementariedad, esta fase se conoce como hibridación; la fase de elongación se presenta a una temperatura que permite la activación de la polimerasa, entre $72-75^{\circ} \mathrm{C}$. En esta etapa, la enzima incorpora los nucleótidos a partir del extremo 3' libre de la región en que han hibridado los cebadores. Este proceso se repite un número determinado hasta obtener un número exponencial de copias del fragmento inicial (Mullis et al., 1986; Pérez de Castro, 2005; Butler, 2015a; Márquez et al., 2016).

Posteriormente, los productos de la PCR, denominados amplicones de STR, deben ser analizados por medio de cromatografías, que pueden ser en gel o capilar (Thermo Fisher, 2014), esta última es la más usada por los laboratorios forenses de Colombia (Instituto Nacional de Medicina Legal y Ciencias Forenses, 2018a). El proceso de electroforesis capilar se basa en la estimulación de moléculas fluorescentes unidas a componentes del ADN amplificado; por medio de un láser de argón son estimuladas y las señales emitidas son detectadas por una cámara de detección CCD (Chang et al., 2011). Posteriormente, las señales detectadas se traducen por medio de un software, que permite la obtención de electroferogramas, que muestran la relación entre la intensidad del fluoróforo con respecto a la región amplificada y, de esa manera, realizar una asignación a cada marcador que se analice; esta interpretación, cálculos estadísticos e informe pericial son realizados por el profesional experto (Applied Biosystems, 2016; Butler, 2015a). Actualmente, se emplean kits multiplex comerciales para el análisis de STR los cuales se pueden amplificar varios STR al mismo tiempo; cada de uno de ellos se elige según las necesidades del investigador (Lien et al., 2015). Los tiempos varían según los protocolos de cada kit comercial que se use, los cuales pueden ser entre 45 y 75 minutos y los costos aproximados entre 20 y 25 dólares. Por su parte, el análisis de los productos de PCR se realiza por medio de electroforesis en gel o electroforesis capilar, cuyo proceso demora aproximadamente entre 45 a 50 minutos con costos entre 2,0 a 3,5 dólares por muestra. Los equipos de electroforesis capilar varían según su generación y el número de capilares que posea: la primera generación contiene un solo capilar; la segunda contiene 4, 8 o 96 capilares y, la tercera generación usa 408 capilares con mejores características técnicas que las anteriores versiones (Butler et al., 2004; Bright et al., 2014). 
La interpretación de los resultados se realiza traduciendo los datos que aún no han sido analizados provenientes del equipo de electroforesis capilar, a través de software especializados que se encargan de realizar la identificación y la asignación de cada uno de los alelos (Applied Biosystems, 2016). El perito forense realizará cálculos estadísticos, con los cuales, y por medio de estudios poblacionales compara los datos para generar un resultado de concordancia entre las muestras analizadas (Just \& Irwin, 2018; Haned, 2010). Toda la información recopilada en el estudio se consigna en un informe pericial, en donde se puede observar por medio de tablas los alelos detectados, los cálculos realizados, las conclusiones de éstos y el soporte científico sobre el cual se basan las técnicas y cálculos realizados (Instituto Nacional de Medicina Legal y Ciencias Forenses, 2012); esta información será observada y valorada por la autoridad que realiza la solicitud.

\section{Casos y Resultados Obtenidos en el Ámbito Forense con Secuenciación Convencional de ADN}

La secuenciación tradicional de ADN ha sido ampliamente utilizada como un factor determinante para la resolución de diferentes delitos; por ejemplo, para la individualización de agresores sexuales (Bernath, 2008). Los métodos de secuenciación de ADN se utilizaban en casos de paternidad y en aquellos relacionados a delitos de inmigración, pero no fue hasta la década de 1980, cuando el profesor universitario Alec Jeffreys concentró esta tecnología en la resolución de dos casos separados, pero con características similares de dos infantes de sexo femenino, las cuales fueron asaltadas sexual y posteriormente asesinadas. Debido al alcance logrado por la secuenciación de ADN, se pudo determinar que ambos hechos habían sido perpetrados por la misma persona, Colin Pitchfork, el cual fue la primera persona condenada con ayuda de evidencia basada en ADN (Kirkland et al., 2009). Otro hecho relevante que favoreció al desarrollo e incorporación de las tecnologías de secuenciación de ADN en ámbitos forenses tempranos fue la identificación del desaparecido comandante nazi Josef Mengele después de 40 años de búsqueda, se creía que había huido a América del sur, y allí había muerto en el mar; la policía tenía una pista del lugar en donde estaba enterrado, pero nunca se había confirmado que en efecto era Mengele. Finalmente, en el año 1985, se extrajo una muestra de ADN de los restos y se usó un análisis reverso de paternidad usando como base para comparar la sangre del hijo de Mengele, lo que resultó en la identificación (Saad, 2005). A partir de estos dos sucesos, el análisis de ADN se convirtió en una de las principales herramientas para la resolución de delitos de individualización de personas (Villalobos Rangel, 2017).

\section{Tiempo y Costos de la Secuenciación Convencional}

La secuenciación tradicional ha sido utilizada desde 1977 para la detección de marcadores de ADN; fue altamente importante en la década de 1990 en el proyecto de secuenciación del genoma humano (Heather \& Chain, 2016). Debido a los procesos involucrados en esta técnica y en los instrumentos necesarios, la secuenciación tradicional ha tenido altos costos, que varían dependiendo de las necesidades y de los laboratorios encargados de realizar este procedimiento; llegando a un costo promedio de 500 dólares por MegabaseMb secuenciada (Rubio et al., 2020). En cuanto al tiempo, este procedimiento está limitado por el volumen de secuenciación, en promedio se puede realizar el análisis con secuenciación tradicional en 30 minutos cada 900 pares de bases secuenciadas. (Chopin, 2012; Causapé et al., 2021).

\section{Secuenciación de Nueva Generación (NGS) en Genética Forense}

Pueden existir situaciones que pueden poner en duda los resultados de un informe pericial y, por consiguiente, la posible decisión jurídica, las cuales se presentan al realizar el análisis de STR por medio de electroforesis capilar. Se puede encontrar productos anómalos o de difícil interpretación posteriores a la amplificación por PCR, como las bandas Stutter, la detección de nuevas mutaciones, alelos nulos y silentes, que podrían alterar el resultado esperado al realizar un cotejo forense (Butler, 2015a; Penacino et al., 2003). Por esta razón, se ha recurrido a la secuenciación de nueva generación o NGS, cambiando completamente los procesos de secuenciación de fragmentos específicos y genomas completos (Yoshinaga et al., 2018). 
La NGS (también conocida como secuenciación masiva en paralelo) es implementada por diferentes casas comerciales en el mundo, de las cuales hay dos que son las más utilizadas y emplean dos enfoques diferentes para generar secuencias de buena calidad en un corto tiempo. La primera de estas plataformas se conoce como lon Torrent ${ }^{\circledR}$, en esta técnica se fragmenta el ADN, y posteriormente se une a perlas insolubles, que están unidas a una laminilla o flow cell. El ADN, que se encuentra en cada perla, presenta una polimerización, en donde la fosforilación ocurrida en este proceso permite la liberación de protones al formar cada enlace covalente de la doble cadena, generando un cambio de $\mathrm{pH}$ en la solución. Así, la unidad automatizada al analizar todos los cambios de $\mathrm{pH}$ producidos por cada nucleótido al polimerizar permite obtener una lectura de la secuencia completa en un tiempo reducido (Perkel, 2010; Biocompare, s.f.).

La segunda técnica es realizada por las plataformas NGS MiSeq y HiSeq, esta se basa en una serie de procesos: 1. Preparación de librerías, para las cuales es necesarias la fragmentación del ADN, amplificación de los fragmentos y la preparación de la biblioteca. 2. Amplificación clonal, esto se realiza en la laminilla o flow cell, donde son capturados los fragmentos de ADN que se encuentran unidos a los adaptadores (diferenciadores entre fragmentos y muestras) y a secuencias universales; en este paso se realiza una gran cantidad de amplificados de las zonas de interés. 3. La polimerización se inicia al unirse los adaptadores con la flow cell que presenta oligonucleótidos que se pueden unir formando un puente; posteriormente, se inicia la secuenciación por síntesis, que se efectúa al agregar base por base en ciclos de secuenciación; en cada uno de ellos se presenta la liberación de un fluorocromo y la señal emitida por cada una de estas moléculas es detectada para su análisis. 4. Finalmente, los datos son estudiados al comparar las secuencias anómalas de las secuencias reales $y$, de esta manera, se obtiene las lecturas de las regiones o fragmentos de interés (Yoshinaga et al., 2018; Illumina, 2016a). Existen diferentes técnicas de NGS con procedimientos similares a las dos anteriores mencionadas, las cuales son ofrecidas por diferentes laboratorios, con distintos equipos utilizados y costos variables del procedimiento. Algunas de estas técnicas son: 454 GS FLX+, GS Junior, SOliD, Retrovolocity, Ion Proton, Ion PGM, SMRT, Heliscope y Nanopore (Kulski, 2015). En la Tabla 1 se evidencian los laboratorios que ofrecen estas técnicas, además de una comparación de los diferentes precios de acuerdo al volumen de secuenciación.

\section{Tabla 1}

Diferentes plataformas NGS con la compañía que lo ofrece, costos en los equipos y costos de acuerdo con el volumen de secuenciación

\begin{tabular}{|c|c|c|c|c|c|}
\hline Plataforma NGS & Compañía & $\begin{array}{c}\text { Lecturas por } \\
\text { ejecución }\end{array}$ & Generación & $\begin{array}{c}\text { Costo en dólares } \\
\text { del equipo }\end{array}$ & $\begin{array}{c}\text { Costo por 10^6 } \\
\text { secuenciaciones } \\
\text { en dólares }\end{array}$ \\
\hline Sanger & Life Technologies & 1 & Primera & 95000 & 2400 \\
\hline 454 GS FLX+ & Roche & $1 \times 10 \wedge 6$ & Segunda & 500000 & 10 \\
\hline GS Junior & Roche & $1 \times 10 \wedge 5$ & Segunda & 100000 & 9 \\
\hline HiSeq & Illumina & $5 \times 10 \wedge 9$ & Segunda & 750000 & 0,1 \\
\hline MiSeq & Illumina & $3 \times 10 \wedge 8$ & Segunda & 125000 & 0,13 \\
\hline Ion Torrent & Thermo Fisher & $5 \times 10 \wedge 5$ & Segunda & 50000 & 0,1 \\
\hline SOliD & Life Technologies & $1 \times 10 \wedge 9$ & Segunda & 350000 & 0,13 \\
\hline Retrovolocity & BGI & $1 \times 10 \wedge 9$ & Segunda & $12 \times 10 \wedge 6$ & 0,01 \\
\hline Ion Proton & Life Technologies & $6 \times 10 \wedge 7$ & Segunda & 215000 & 1 \\
\hline Ion PGM & Life Technologies & $1 \times 10 \wedge 6$ & Tercera & 80000 & 1 \\
\hline SMRT & Pac Bio & $7 \times 10 \wedge 9$ & Tercera & $1.35 \times 10 \wedge 6$ & 2 \\
\hline
\end{tabular}




\begin{tabular}{|c|c|c|c|c|c|}
\hline Plataforma NGS & Compañía & $\begin{array}{c}\text { Lecturas por } \\
\text { ejecución }\end{array}$ & Generación & $\begin{array}{c}\text { Costo en dólares } \\
\text { del equipo }\end{array}$ & $\begin{array}{c}\text { Costo por 10^6 } \\
\text { secuenciaciones } \\
\text { en dólares }\end{array}$ \\
\hline Heliscope & Helicos & $6 \times 10 \wedge 4$ & Tercera & $\underline{1000}$ & 0,01 \\
\hline Nanopore & Oxford & & Tercera & $1 \times 10 \wedge 6$ & $<1$ \\
\hline
\end{tabular}

Nota. El genoma humano posee 3×109 bp o 3 Gb (Cervantes-García et al., 2005).

En las últimas décadas, las técnicas de secuenciación han avanzado con gran rapidez y precisión, esto ha permitido que se explore su incorporación en diferentes ámbitos de las ciencias forenses (ÁlvarezCubero et al., 2017). Una de las principales razones por las cuales las técnicas de secuenciación de nueva generación han cobrado importancia en esta área de investigación es por su utilidad para generar una considerable cantidad de información, incluso cuando la presencia de ADN es escasa; es importante destacar que en los casos que involucran las ciencias forenses, normalmente la cantidad de muestra disponible para análisis es insuficiente 0 está en un estado degradado (Just \& Irwin, 2018). La secuenciación de nueva generación aporta entonces, la posibilidad de realizar un análisis de ADN autosómico, ADN mitocondrial y marcadores cromosómicos $X$ e $Y$ con limitada cantidad de muestra, esta característica permite que la secuenciación de nueva generación sea considerada como una herramienta indispensable para la individualización de personas en desastres naturales o eventos donde las muestras sean escasas o estén contaminadas (Álvarez-Cubero et al., 2017).

De otro lado, la información obtenida a través de esta metodología permite obtener datos robustos de importante utilidad cuando se pretende realizar un mapeo de variación epigenética. Su aporte tiene utilidad en casos donde no se tiene ningún sospechoso identificado o relacionado, puesto que las NGS permiten realizar una estimación del fenotipo del ADN, permitiendo que en una muestra se identifique una perfilación racial o los rasgos físicos (Hunter, 2018).

\section{Casos y Resultados en el Ámbito Forense Obtenidos a través de la Secuenciación de Nueva Generación (NGS)}

El desarrollo de la técnica de NGS, se caracteriza por la confiabilidad, rapidez y precisión en la obtención del resultado; flexibilidad, robustez, resolución de OL (off ladder o alelos no presentes en la escala de lectura), detección de nuevas mutaciones, especificidad, sensibilidad y bajo costo (Wu et al., 2019), en comparación con los métodos STR utilizados convencionalmente. Esto ocurre, dado que los polimorfismos en secuencia tienen un mayor poder de discriminación estadística y se puede obtener un mayor potencial discriminatorio, al analiza los polimorfismos de cada marcador genético (Guo et al., 2016). La precisión de la secuenciación de nueva generación ha permitido su aplicación en ámbitos forenses, específicamente en casos que involucran métodos genéticos de individualización; sin embargo, como se trata de un conjunto de técnicas relativamente modernas, no se evidencian muchos casos específicos resueltos con el uso de esta metodología (Aly \& Sabri, 2015; de Knijff, 2019).

A continuación, se resaltan algunos casos que se utilizan como base para constatar la relevancia que tendría la implementación de técnicas de secuenciación de nueva generación en el mundo:

1. Caso de dos menores de edad de sexo femenino que fueron accedidas carnalmente por dos hombres en la ciudad metropolitana de Haryana, India; específicamente en este caso, se obtuvieron muestras de semen en la ropa interior de una de las menores y con el uso del sistema genómico forense MiSeq FGxTM se generaron perfiles de ADN, resultando en la identificación de diferentes marcadores autosómicos, SNP de identidad, ascendencia y fenotipos amplificados. En este caso, las tecnologías de nueva generación proporcionaron más información que la técnica tradicional de electroforesis capilar, puesto que con esta última solo se pueden analizar a la vez pocos lugares donde se encuentran ubicados los genes dentro de los cromosomas o Loci, impidiendo la obtención de información sobre 
genealogía y fenotipos. Por lo anterior, se pudo vincular formalmente a uno de los sospechosos al caso como resultado de la información fenotípica que solo pudo ser obtenida mediante la secuenciación de nueva generación (Kawatra et al., 2014).

2. Una mujer de 28 años fue asaltada sexualmente en 2015 en Los Países Bajos; después del suceso, se recolectó evidencia física y elementos materiales probatorios de su ropa y de partes de su cuerpo, los cuales fueron enviados al laboratorio forense respectivo. Se procedió a realizar análisis de ADN, empleando electroforesis capilar convencional y, en el 2016 los resultados obtenidos fueron controvertidos en juicio por la defensa del sospechoso debido a que consideraron que existían irregularidades. El análisis de un laboratorio de la contraparte dictaminó que en la muestra había rastros de ADN mayoritariamente de la víctima y menos de un $10 \%$ de naturaleza desconocida, que podría estar relacionado al ADN del perpetrador, pero al estar mezclado junto al ADN de la víctima, la electroforesis capilar no podía aportar más información. El sospechoso fue puesto en libertad; la fiscalía presentó una apelación y se optó por realizar el análisis de nuevo, utilizando técnicas de secuenciación de nueva generación. Con este nuevo análisis se obtuvo mayor cantidad de información debido a que el ADN amplificado y secuenciado mostró que la muestra presentaba un marcador específico masculino, que corresponde al Y-STR, el cual fue aislado y analizado con el ADN del sospechoso, determinándose su coincidencia; estos nuevos resultados llevaron finalmente a que el sospechoso fuera declarado culpable el 17 de enero de 2018 (Honda, 1999; de Knijff, 2019).

\section{Tiempo y Costos Requeridos para la Ejecución de la Secuenciación de Nueva Generación (NGS)}

A través de la tecnología NGS, se tiene el potencial de analizar secuencias específicas con una mayor velocidad a un costo reducido; las casas comerciales que proveen los kits de aplicación forense, como Forenseq y HID-Ion Ampliseq Identity Panel, presentan costos de ejecución menores a un dólar por Megabase secuenciada (Churchill et al., 2016). El tiempo estimado para la construcción de librerías es de aproximadamente 220 minutos (Illumina,
2015; Laurent et al., 2017), y el requerido para el procesamiento de una muestra puede variar según el protocolo seleccionado, registrándose entre 45 a 60 minutos; la capacidad máxima del Flow Cell es de 96 muestras, las cuales se pueden procesar al mismo tiempo (Illumina, 2016; Applied Biosystems, 2016). El análisis de los resultados posteriores a la secuenciación debe ser procesado por medio de softwares de bioinformática dispuestos para esta labor como: Molecular Evolutionary Genetics Analysis (MEGA), Clustal, BLAST, entre otros (Jäger et al., 2017; Illumina, 2016b; 2016c).

Además del costo reducido de las técnicas de NGS, comparado con las técnicas tradicionales, la implementación de esta no requiere de equipos adicionales al secuenciador; la preparación de muestras se realiza con equipamiento estándar de laboratorio (termociclador, centrífuga y pipetas) (Illumina Inc., 2017). Adicionalmente, el modo de operar y trabajar las tecnologías de secuenciación de nueva generación consta de estándares de biología molecular, utilizados en técnicas de secuenciación tradicional, por lo que no es necesario que se realice una capacitación adicional al personal en los laboratorios acreditados en el área forense y, por consiguiente, no genera costos adicionales a este respecto (Battelle, 2011).

\section{Desafíos de la Genética Forense en Colombia frente a las Nuevas Tecnologías}

La Ley 75 de 1968 con la cual se dictaron las normas referentes a las filiaciones en el país, debió ser modificada con la Ley 721 de 2001, teniendo en cuenta los avances científicos en el mundo. Con esta nueva normativa, se introdujo el estudio del ADN como una herramienta de filiación, y posteriormente, con la Ley 906 de 2004 se describe la importancia del ADN como método de identificación forense en el país dentro los sistemas de individualización usados. Por su parte, la acreditación y la certificación nacional de pruebas genéticas para filiaciones, se hace una vez al año a través del Organismo Nacional de Acreditación de Colombia (ONAC), responsable de la acreditación y de la certificación de laboratorios con sujeción a los estándares internacionales establecidos para pruebas de filiación, a través de su área de acreditación de laboratorios de calibración y ensayos (Organismo Nacional de Acreditación de Colombia (ONAC), 2021). 
Si se realiza un análisis detallado de los informes de gestión, presentados en los últimos años dentro del CTI de la Fiscalía General de la Nación y del Instituto Nacional de Medicina Legal y Ciencias Forenses, se encuentra que en el último informe, las áreas encargadas de la identificación humana, como los laboratorios de antropología y genética, presentan un crecimiento paulatino en términos de procesamientos de muestras; donde el laboratorio de genética forense presentó un incremento del $11 \%$ en el año 2020, cada año las muestras procesadas van en aumento (ICMP, 2008; Instituto Nacional de Medicina Legal y Ciencias Forenses, 2020). Lo anterior, indica un aumento en la necesidad de los servicios prestados y en las labores de identificación forense por parte de los laboratorios de genética en el país destinados para este fin. De esta manera, se corrobora los esfuerzos institucionales en los procesos de identificación de individuos generados por el conflicto como parte de las políticas de reparación a las víctimas (Ley 1448 de 2011), como son aquellos provenientes del programa de Justicia y Paz (Arango y Camargo, 2015), la identificación de personas desaparecidas del palacio de justicia (I/A Court H.R., 2014) y actualmente los que se generan del proceso de paz con los grupos armados en el contexto del conflicto armado, como son paramilitares, guerrillas y bandas criminales, en el marco de la Jurisdicción Especial para la Paz (Presidencia de la República \& FARC-EP, 2016; Jurisdicción Especial para la Paz (JEP), 2020).

La información resultante de los procesos de identificación por ADN es consignada en bases de datos de información genética; una de ellas fue implementada en Colombia desde el año 2005 con la adquisición de la plataforma CODIS, propiedad de la agencia estadounidense $\mathrm{FBI}$, que se denominó Base Nacional de Perfiles Genéticos de Aplicación en Investigación Judicial. Esta plataforma es actualizada con información proveniente de los laboratorios de genética del Instituto Nacional de Medicina Legal y Ciencias Forenses de las ciudades de Bogotá, Cali, Medellín y Villavicencio; así como por los laboratorios de genética del CTI de la Fiscalía General de la Nación y de la DIJIN de la Policía Nacional de Colombia (Instituto Nacional de Medicina Legal y Ciencias Forenses, 2009; 2020). Hasta hace pocos años, se encontró que el CODIS almacenó aproximadamente 27.000 perfiles genéticos, de los cuales 2.000 correspondieron a restos humanos en la categoría de desaparecidos y 25.000 a familiares. Empero, el CODIS permanece en constante actualización (Arango y Camargo, 2015; Instituto Nacional de Medicina Legal y Ciencias Forenses, 2020).

Existen módulos específicos para el desarrollo de las investigaciones en este campo como: 1. Módulos de criminalística y vinculados judicialmente; posible parentesco de restos óseos con una familia, que se evalúan a través de los módulos. 2. Restos óseos. 3. Módulo de familias vinculadas. El administrador de la base de datos realiza el reporte e informa a las áreas forenses del Instituto Nacional de Medicina Legal y Ciencias Forenses como patología, antropología o Red Nacional de Personas Desaparecidas, quienes analizan e intercambian información para verificar la coincidencia genética (Instituto Nacional de Medicina Legal y Ciencias Forenses, 2018b; 2020). Al observar el crecimiento de los servicios en los laboratorios de genética del INMYCF, se puede realizar una estimación a 2021 al analizar los datos presentados por los informes de gestión institucionales entre los años 2014 a 2020 proyectando un aumento del 7,5\% anual, en la cantidad de muestras analizadas, las cuales en su gran mayoría provienen de procesos de identificación de restos óseos (Instituto Nacional de Medicina Legal y Ciencias Forenses, 2017; 2015; 2018b; 2020).

\section{Discusión y Conclusión}

Teniendo en cuenta la realidad técnico-científica. en cuanto al manejo de los laboratorios de genética forense y su papel como herramienta para la justicia, se puede concluir que las técnicas genéticas disponibles en la investigación forense con bases moleculares han permitido un adelanto importante en la investigación en lo referente a análisis de muestras forenses biológicas de interés criminal y/o filiación, aportando significativamente a la resolución de casos; debido a la gradual adición de marcadores genéticos, con lo que se ha logrado desarrollar la técnica de NGS que admite rastreo 
de hasta 230 marcadores genéticos, aportando resultados más efectivos y confiables (Jäger et al., 2017; Sharma et al., 2017; Xavier \& Parson, 2017; Illumina, 2016b). Se observa entonces que hoy en día el uso de técnicas moleculares como NGS permiten el análisis de un genoma aún más completo, secuencias determinadas de ADN, largas o cortas para detectar y analizar fragmentos de interés para la investigación en genética forense, lo cual se convierte en una herramienta eficiente para la resolución de casos.

Aunque las nuevas tecnologías tienen ventajas, también presentan algunos inconvenientes, como los generados por los inhibidores de la amplificación como el calcio y la hematina con el módulo de trabajo Ion AmpliSeq a diferencia del análisis con el módulo Forenseq; inhibidores como la melanina y el colágeno son más susceptibles al analizar con el módulo Forenseq que con el Ion AmpliSeq. De igual manera, los amplicones de gran tamaño son menos sensibles a inhibidores que aquellos de menor tamaño (Elwick et al., 2018; Fattorini et al., 2017). La capacidad analítica del sistema NGS se mejoraría aún más si se incluyeran más loci con variantes de secuencia en el sistema de detección; ForenSeq UAS no analiza secuencias de flanqueo de todos los

\section{Referencias}

Afrifah, K., Badu-Boateng, A., Antwi-Akomeah, S., Motey, E., Boampong, E., ... Twumasi, P. (2020). Forensic identification of missing persons using DNA from surviving relatives and femur bone retrieved from salty environment. Journal of Forensic Science and Medicine, 6(1), 40-44. DOI: https://doi.org/10.4103/jfsm.jfsm

Alejos, P., Aragón, M., y Romero, A. (2008). Extracción y purificación de ADN. En A. Cornejo, A. Serrato, B. Rendón, y M. Rocha (Comp.). Herramientas moleculares aplicadas en ecología. (pp. 1-26). SEMARNAT, INECC, UAM-I.

Álvarez-Cubero, M., Saiz, M., Martínez-García, B., Sayalero, S., Entrala, C., Lorente, J., \& Martínez-González, L. (2017). Next generation sequencing: an application in forensic sciences?. Annals of Human Biology, 44(7), 581-592. DOI: 10.1080/03014460.2017.1375155 loci; por lo tanto, algunos alelos polimórficos en las secuencias flanqueantes (Wu et al., 2019).

En cuanto al flujo de datos, el gran volumen de información producida al mismo tiempo, puede ser una barrera para el control de los resultados obtenidos por NGS, ya que su interpretación por el personal experto puede ser largo y dispendioso. Por esta razón, una fracción significativa del personal pericial estaría encargado de los procesos analíticos, en lugar de llevar a cabo procesos estrictamente técnicos en los laboratorios (Zaragoza et al., 2010).

En Colombia, el análisis de nueva generación podría tener una contribución inmediata y accesible para el desarrollo de la genética forense, permitiendo a los peritos expertos realizar su labor con mayor eficiencia, teniendo la capacidad de obtener resultados positivos al analizar ciertas secuencias en muestras que por métodos convencionales fueron negativas (Marshall et al., 2017); evitando de cierta manera los reprocesos y encaminando su experticia en el análisis e interpretación de los datos, con el objetivo de generar informes de resultados de calidad a las autoridades, contribuir a una menor tasa de impunidad, y a una mayor reparación de las víctimas de la violencia por el conflicto histórico en nuestra nación.
Alvis-Arango, A. (2016). Evaluación de los métodos fenol-cloroformo y columnas de sílice para extracción de $A D N$ a partir de tejido óseo. Colombia Forense, 2(1), 69-74. DOl: https://doi. org/10.16925/cf.v3i1.1199

Aly, S., \& Sabri, D. (2015). Next generation sequencing (NGS): a golden tool in forensic toolkit. Archiwum Medycyny Sądowej i Kryminologii/ Archives of Forensic Medicine and Criminology, 65(4), 260-271. DOl: https://doi.org/10.5114/ amsik.2015.61029

Applied Biosystems. (2016). New features and changes in GeneMapper ID-X Software. https:// assets.thermofisher.com/TFS-Assets/LSG/ manuals/4477684B.pdf

Arango, J., y Camargo, M. (2015). Proceso de identificación de cadáveres en el marco del proyecto de Justicia y Paz en el Laboratorio 
de Genética Forense del Instituto Nacional de Medicina Legal y Ciencias Forenses, Regional Suroccidente, entre octubre 17 del 2008 y junio 21 del 2012.

Bernath, V. (2008). El ADN como herramienta para la resolución de procesos judiciales. Pasado, presente y futuro. Química Viva, 7(2), 103-112.

Biesecker, L., Bailey-Wilson, J., Ballantyne, J., Baum, J., Bieber, F., Brenner, C., ... Walsh, A. (2005). DNA Identifications After the 9/11 World Trade Center Attack. Policy Forum. Epidemiology, 310(5751), 1122-1123. DOI: https://doi.org/10.1126/ science.1116608

Biocompare. (s.f.). Ion personal genome machine system from thermo fisher scientific. https:// www.biocompare.com/23967-Next-GenerationSequencers/11182075-Ion-Personal-GenomeMachine-System/

Bright, J., Neville, S., Curran, J., \& Buckleton, J. (2014). Variability of mixed DNA profiles separated on a 3130 and 3500 capillary electrophoresis instrument. Australian Journal of Forensic Sciences, 46(3), 304-312. DOI: https:// doi.org/10.1080/00450618.2013.851279

Brito, F., Nunes, M., Prata, D., Martha, S., Bottino, C., \& Garrido, R. (2019). DNA extraction of urinary bladder swabs collected from carbonized and decomposing corpses: possible application in disaster victim identification. Legal Medicine, 37, 15-17. DOI: https://doi.org/10.1016/j. legalmed.2018.12.002

Butler, J. (2015a). Advanced topics in forensic DNA typing- Interpretation. Academic Press.

Butler, J. (2015b). U.S. initiatives to strengthen forensic science \& international standards in forensic DNA. Forensic Science International: Genetics, 18, 4-20. DOl: https://doi.org/10.1016/j. fsigen.2015.06.008

Butler, J., Buel, E., Crivellente, F., \& McCord, B. (2004). Forensic DNA typing by capillary electrophoresis using the $\mathrm{ABI}$ Prism 310 and 3100 genetic analyzers for STR analysis. Electrophoresis, 25(1011), 1397-1412.

Causapé, C., González Candelas, F., Carmona, M., y Palomo, A. (2021). Procedimientos en Microbiología Clínica. Sociedad Española de Enfermedades Infecciosas y Microbiología Clínica (SEIMC).
Cervantes-García, D., González-Ruíz, C., y MayekPérez, N. (2005). Proyecto Genoma Humano: Situación actual y perspectivas. Investigación y Ciencia, 13(33), 56-63. https://www.redalyc.org/ pdf/674/67403309.pdf

Chang, C., Maloof, J., \& Wu, S. (2011). COP1Mediated Degradation of BBX22/LZF1 Optimizes Seedling Development in Arabidopsis. Plant Physiology, 156(1), 1-10. DOl: https://doi. org/10.1104/pp.111.175042

Chopin, M. (2012). Principios básicos de electroforesis capilar: Técnica analítica de separación de analitos. Revista Tecnología y Salud, 1(2), 86-89. https://www.medigraphic.com/pdfs/invdis/ir2012/ir122g.pdf

Churchill, J., Schmedes, S., King, J., \& Budowle, B. (2016). Evaluation of the Illumina ${ }^{\circledR}$ Beta Version ForenSeqTM DNA Signature Prep Kit for use in genetic profiling. Forensic Science International: Genetics, 20, 20-29. DOl: https:// doi.org/10.1016/j.fsigen.2015.09.009

de Armas, Y., Capó, V., López, L.., Mederos, L., \& Díaz, R. (2011). Comparación de tres métodos de extracción de ADN de tejidos embebidos en parafina. Biotecnología Aplicada, 28(1), 44-47. https://www.medigraphic.com/cgi-bin/new/ resumen.cgi? IDARTICULO=36399

de Knijff, P. (2019). How Next Generation Sequencing Resolved a Difficult Case, Leading to the First Criminal Conviction of its Kind. Verogen, 1-4. https:// cdn2. hubspot. net/ hubfs/ 6058606/ Verogen- First- NGS- Court- Case- Study_Final_ VD2019024_8.5x11- web.pdf?_ hstc $=2386$ 09695. bed7 4b81c f4041 e42ad ad16833 ab858 4.1576870704888.1576870704888.157687070 4888.1\& hssc $=238609695.1 .1576870704888$

DNeasy blood \& tissue handbook. (2006). For purification of total DNA from animal blood, animal tissue, rodent tails, ear punches, cultured cells, fixed tissue, bacteria, insects. QiaGen.

Elwick, K., Zeng, X., King, J., Budowle, B., \& HughesStamm, S. (2018). Comparative tolerance of two massively parallel sequencing systems to common PCR inhibitors. International Journal of Legal Medicine, 132, 983-995. DOI: https://doi. org/10.1007/s00414-017-1693-4

Fattorini, P., Previderé, C., Carboni, I., Marrubini, G., Sorçaburu-Cigliero, S., Grignani, P., \& Ricci, U. (2017). Performance of the ForenSeqTM DNA 
Signature Prep kit on highly degraded samples. Electrophoresis, 38(8), 1163-1174. DOI: https:// doi.org/10.1002/elps.201600290

García García, R., Oriana, O., Lagares, A., Varela, L., y Nelson. (2008). Utilización de resina Chelex en la extracción de ADN de varios tipos de tejidos de la tortuga marina Caretta caretta, para la amplificación de marcadores moleculares. En El Caribe en las ciencias básicas e ingeniería (pp. 343-354). Universidad del Atlántico.

García-Aceves, M., Romero Rentería, O., DíazNavarro, X., \& Rangel-Villalobos, H. (2008). Paternity tests in Mexico: Results obtained in 3005 cases. Journal of Forensic and Legal Medicine, 55, 1-7. DOl: https://doi.org/10.1016/j. jflm.2018.02.003

Grupo Español y Portugués de la ISFG. (s.f.). Guía para implementar un sistema de calidad en los laboratorios de genética forense. https://www. yumpu.com/es/document/read/32140755/guaa-para-implementar-un-sistema-de-calidad-enlos-gep-isfg

Guo, F., Zhou, Y., Liu, F., Yu, J., Song, H., Shen, H., ... Jiang, X. (2016). Evaluation of the early access STR Kit vl on the lon Torrent PGMTM platform. Forensic Science International: Genetics, 23, 111-120. DOI: https://doi.org/10.1016/j. fsigen.2016.04.004

Haned, H. (2010). Evaluation of statistical methods for the analysis of forensic DNA mixtures. To cite this version. Université Claude Bernard. https://tel.archives-ouvertes.fr/tel-00817181/ document

Heather, J., \& Chain, B. (2016). The sequence of sequencers: The history of sequencing DNA. Genomics, 107(1), 1-8.

Honda K., Roewer, L., \& de Knijff, P. (1999). Male DNA typing from 25-year-old vaginal swabs using $Y$ chromosomal STR polymorphisms in a retrial request case. Journal of Forensic Sciences, 44(4), 868-872.

Hunter, P. (2018). Uncharted waters: Nextgeneration sequencing and machine learning software allow forensic science to expand into phenotype prediction from DNA samples. EMBO Reports, 19(3), e45810. DOl: https://doi. org/10.15252/embr.201845810

I/A Court H.R. (2014). Case of Rodríguez Vera et al. (The Disappeared from the Palace of Justice) v. Colombia (Preliminary Objections, Merits, Reparations and Costs). Series C N(1). http:// www.corteidh.or.cr/docs/casos/articulos/ seriec_287_ing.pdf

Ibarra, A. (2015). Estudio de marcadores genéticos bialélicos para aplicaciones forenses: SNPs autosómicos e Indels específicos de cromosoma X [Tesis de doctorado, Universidad de Antioquia]. https://bibliotecadigital. udea.edu.co/bitstream/10495/3093/1/ I b a r r a R o d rig u e z A _ 2015 _ estudioMarcadoresGeneticos.pdf

ICMP. (2008). Informe De Icmp: Respuesta de Colombia a las desapariciones forzadas.

Illumina. (2015). Signature Prep.

Illumina. (2016a). An introduction to Next-Generation Sequencing Technology. www.illumina.com/ technology/next-generation-sequencing.html

Illumina. (2016b). ForenSeq TM Universal Analysis Software Guide. (August).

Illumina. (2016c). Nextera ${ }^{\circledR}$ Rapid Capture Enrichment Reference Guide. https://support. illumina.com/content/dam/illumina-support/ documents/documentation/chemistry_ documentation/samplepreps_nextera/ nexterarapidcapture/nextera-rapid-captureenrichment-guide-15037436-01.pdf

Illumina. (2017). Illumina sequencing introduction. Illumina Sequencing Introduction, October, 1-8. https://www.illumina.com/documents/ products/illumina_sequencing_introduction.pdf

Instituto Nacional de Medicina Legal y Ciencias Forenses. (2009). Ingreso de perfiles genéticos a la base nacional de datos de perfiles genéticos de aplicación en investigación judicial.

Instituto Nacional de Medicina Legal y Ciencias Forenses. (2012). Estandarizados de Trabajo pets vigentes a 2012 relacionados con el Proceso de Identificación Genética mediante el establecimiento de relaciones de parentesco.

Instituto Nacional de Medicina Legal y Ciencias Forenses. (2018a). Certificación de laboratorios de genetica 2018 bajo la ISO 17025:2055.

Instituto Nacional de Medicina Legal y Ciencias Forenses. (2018b). Portafolio de Servicios. http://esehospitalsanmarcoschinchina-caldas.gov.co/apc-aa-fil es/65393163643538633135393339653936/ portafolio-virtual-hsm-ok.pdf 
Instituto Nacional de Medicina Legal y Ciencias Forenses. (2020). Informe de Gestión 2020. https://www.medicinalegal. gov.co/documents/20143/39839/ Informe+de+Gestion+2020.pdf

Iwamura, E., Guimarães, M., \& Evison, M. (2016). DNA methods to identify missing persons. En S. Morewitz, \& Sturdy, C. Handbook of Missing Persons (pp. 337-352). Springer. DOl: https://doi. org/10.1007/978-3-319-40199-7_22

Jäger, A., Alvarez, M., Davis, C., Guzmán, E., Han, Y., Way, L., ... Holt, C. (2017). Developmental validation of the MiSeq FGx Forensic Genomics System for Targeted Next Generation Sequencing in Forensic DNA Casework and Database Laboratories. Forensic Science International: Genetics, 28, 52-70. DOI: https:// doi.org/10.1016/j.fsigen.2017.01.011

Jurisdicción Especial para la Paz (JEP). (2020). Informe Estadistico. https://www.jep.gov. co/rendiciondecuentas/Informes\%202020/ Informe\%20Estadi\%CC\%81stico.pdf

Just, R., \& Irwin, J. (2018). Use of the LUS in sequence allele designations to facilitate probabilistic genotyping of NGS-based STR typing results. Forensic Science International: Genetics, 34, 197-205. DOl: https://doi.org/10.1016/j. fsigen.2018.02.016

Katsanis, S. \& Wagner, J. (2012). Characterization of the Standard and Recommended CODIS Markers. Journal of Forensic Sciences, 58(1), 169-172. DOl: https://doi.org/10.1111/j.15564029.2012.02253.x

Kawatra, R., Rai, A., Rana, K., \& Sharma, R. (2014). Investigation of rape case by using NGS based MiSeq FGx forensic genomics system - a case of deleted amelogenin male. https:// vb6ykw2twb15uf9341ls5n11-wpengine.netdnassl.com/wp-content/uploads/2019/08/70-Dr.Rajeev-Kawatra.pdf

Kesseli, R., Ochoa, O., \& Michelmore, R. (1991). Variation at RFLP loci in Lactuca spp. and origin of cultivated lettuce (L. sativa). Genome, 34(3), 430-436

Kirkland, J., Reddy, A., \& Khushbu P. (2009). DNA Fingerprinting. Worcester Polytechnic Institute. https://web.wpi.edu/Pubs/E-project/Available/ E-project-082809-134907/unrestricted/IQP.pdf
Kulski, J. (2016). Next-Generation sequencing an overview of the history, tools, and "omic" applications. IntechOpen. DOI: https://doi.org/ http://dx.doi.org/10.5772/61964

Laurent, F., Ausset, L., Clot, M., Jullien, S., Chantrel, Y., Hollard, C., \& Pene, L. (2017). Automation of library preparation using Illumina ForenSeq kit for routine sequencing of casework samples. Forensic Science International: Genetics Supplement Series. DOl: https://doi. org/10.1016/j.fsigss.2017.09.156

Ley 75 de 1968. Por la cual se dictan normas sobre filiación y se crea el Instituto Colombiano de Bienestar Familiar. Congreso de la República. 30 de diciembre de 1968. DO 32682.

Ley 906 de 2004. Por la cual se expide el Código de Procedimiento Penal. (Corregida de conformidad con el Decreto 2770 de 2004). Congreso de la República. 31 de agosto de 2004. DO 45657.

Ley 1408 de 2010. Por la cual se rinde homenaje a las víctimas del delito de desaparición forzada y se dictan medidas para su localización e identificación. Congreso de Colombia. 20 de agosto de 2010. DO 47807.

Ley 1448 de 2011. Por la cual se dictan medidas de atención, asistencia y reparación integral a las víctimas del conflicto armado interno y se dictan otras disposiciones. Congreso de la República. 10 de junio de 2011. DO 48096.

Ley 721 de 2001. Por medio de la cual se modifica la Ley 75 de 1968. Congreso de la República. 24 de diciembre de 2001. DO 44661.

Lien, E. Y., Leader, T., \& Dna, N. (2015). Forensic biology protocols for forensic STR analysis Approving Authority. https://wwwl.nyc.gov/ assets/ocme/downloads/pdf/technicalmanuals/forensic-biology-technical-manuals/ protocols_for_str_analysis_050115.pdf.

Márquez, M., Concepción, J., González-Marcano, E., \& Mondolfi, A. (2016). Detection of Trypanosoma cruzi by polymerase chain reaction. En R. Luthra, R. Singh, \& K. Patel. Clinical applications of PCR (pp. 125-141). Springer. DOI: https://doi. org/10.1007/978-1-4939-3360-0

Marshall, C., Sturk-Andreaggi, K., DanielsHigginbotham, J., Oliver, R., Barritt-Ross, S., \& McMahon, T. (2017). Performance evaluation of a mitogenome capture and Illumina sequencing protocol using non-probative, case-type 
skeletal samples: Implications for the use of a positive control in a next-generation sequencing procedure. Forensic Science International: Genetics, 31, 198-206. DOl: https://doi. org/10.1016/j.fsigen.2017.09.001

Mccord, B. (2018). Rapid and selective extraction of male DNA from rape kits and other forensic evidence using pressure cycling, National Criminal Justice Reference Service, 1-14. https:// www.ojp.gov/pdffiles1/nij/grants/251801.pdf

Mullis, K., Horn, G., Saiki, R., Erlich, H., Faloona, F., \& Scharf, S. (1986). Specific enzymatic amplification of DNA in vitro: the polymerase chain reaction. Cold Spring Harb Symp Quant Bio, 51, 263-273. DOl: https://doi.org/10.1101/ sqb.1986.051.01.032

Organismo Nacional de Acreditación de Colombia (ONAC). (2021). ONAC. https://onac.org.co/ presentacion

Paredes, M., y Romero, R. (2009). Genética forense en la identificación de cadaveres. En M., Morales, E., Niño. Identificación de cadáveres en la práctica forense (pp. 125-150). Instituto Nacional de Medicina Legal y Ciencias Forenses.

Penacino, G. (2003). Análisis de ADN: ¿Errores técnicos o manipulación de resultados? http:// slagf.org.ar/a2/000000076.pdf

Penacino, G., Sala, A., \& Corach, D. (2003). Are DNA tests infallible? International Congress Series, 1239, 873-877. DOl: https://doi.org/10.1016/ S0531-5131(02)00558-7

Pérez de Castro, A. (2005). Reacción en cadena de la polimerasa (Polymerase Chain Reaction, PCR). Universidad Politécnica de Valencia.

Perkel, J. (2010). Making Contact with Sequencing' s Fourth Generation. BioTechniques, 50(2), 9395. DOl: https://doi.org/10.2144/000113608

Presidencia de la República, \& FARC-EP. (2016). Que como resultado de los diálogos exploratorios referidos se produjo un acuerdo final para la terminación del conflicto y da construcción de una paz estable y duradera, 1-310. http://www.altocomisionadoparalapaz. gov.co/procesosyconversaciones/ Documentoscompartidos/24-112016NuevoAcuerdoFinal.pdf

QiaGen. (2017). Investigator. Quantiplex Pro Handbook. For quantification of human and male DNA in forensic samples. QiaGen.
QiaGen. (2018). QIAxpert User Manual. (February). QuiaGen.

Rees, B., \& Rothwell, T. (1975). The identification of phosphoglucomutase isoenzymes in semen stains and its use in forensic casework investigation. Medicine, Science and the Law, 15(4), 284-293. DOl: https://doi. org/10.1177/002580247501500411

Rubio, S., Pacheco-Orozco, R., Gómez, A., Perdomo, S., \& García-Robles, R. (2020). Secuenciación de nueva generación (NGS) de ADN: presente y futuro en la práctica clínica. Universitas Médica, 61(2), 1.15. DOl: https://doi.org/10.11144/ javeriana.umed61-2.sngs

Saad, R. (2005). Discovery, development, and current applications of Dna identity testing. Baylor University Medical Center Proceedings, 18(2), 130-133. DOl: https://doi.org/10.1080/089 98280.2005.11928051

Saiz, Belén Martínez-García, Sara M. Sayalero, Carmen Entrala, Jose Antonio Lorente \& Luis Javier Martinez-Gonzalez (2017) Next generation sequencing: an application in forensic sciences?. Annals of Human Biology, 44(7), 581-592. DOl: 10.1080/03014460.2017.1375155

Sharma, V., Chow, H., Siegel, D., \& Wurmbach, E. (2017). Qualitative and quantitative assessment of Illumina's forensic STR and SNP kits on MiSeq FGxTM. PLOS ONE, 12(11), 1-21. DOI: https://doi. org/10.1371/journal.pone.0187932

Sudoyo, H., Widodo, P. T., Suryadi, H., Lie, Y. S., Safari, D., Widjajanto, A., ... Marzuki, S. (2008). DNA analysis in perpetrator identification of terrorism-related disaster: Suicide bombing of the Australian Embassy in Jakarta 2004. Forensic Science International: Genetics, 2(3), 231-237. DOl: https://doi.org/10.1016/j.fsigen.2007.12.007

ThermoFisher Scientific. (2014). Fragment-AnalysisChemistry-Guide. https://www.thermofisher. com/content/dam/LifeTech/global/Forms/PDF/ fragment-analysis-chemistry-guide.pdf

ThermoFisher Scientific. (2000). NanoDrop 2000 / 2000c Spectrophotometer. https://www. thermofisher.com/order/catalog/product/ ND2000CLAPTOP

Tozzo, P., Ponzano, E., Spigarolo, G., Nespeca, P., \& Caenazzo, L. (2018). Collecting sexual assault history and forensic evidence from adult women in the emergency department: a 
retrospective study, 18(383), 1-6. https://www. ncbi.nlm.nih.gov/pmc/articles/PMC5975504/ pdf/12913_2018_Article_3205.pdf

Villalobos Rangel, H. (2017). Las pruebas de ADN, cuantificacion. Ciencias Forenses de Honduras, 3(2), 28-38.

Watkins, W. (1980). Biochemistry and genetics of the ABO, Lewis, and $P$ blood group systems. Advances in Human Genetics, 10, 1-136, 379-385. http://www.ncbi.nlm.nih.gov/pubmed/6156588

Watson, J., Baker, T., Bell, S., Gann, A., Levine, M., \& Losick, R. (2007). Molecular Biology of the Gene. Pearson.

Wu, J., Li, J., Wang, M., Li, J., Zhao, Z., Wang, Q., ... Deng, Y.. (2019). Evaluation of the MiSeq FGx system for use in forensic casework. International Journal of Legal Medicine, 133, 689-697. DOl: https://doi.org/10.1007/s00414-018-01987-x
Xavier, C., \& Parson, W. (2017). Evaluation of the Illumina ForenSeqTM DNA Signature Prep Kit - MPS forensic application for the MiSeq FGxTM benchtop sequencer. Forensic Science International: Genetics, 28, 188-194. DOl: https:// doi.org/10.1016/j.fsigen.2017.02.018

Yoshinaga, Y., Daum, C., He, G., \& O'Malley, R. (2018). Genome sequencing. En R. de Vries, A. Tsang, I. Grigoriev. Methods in Molecular Biology (pp. 3752). Springer. DOI: https://doi.org/10.1007/9781-4939-7804-5_4

Zaragoza, M., Fass, J., Diegoli, M., Lin, D., \& Arbustini, E. (2010). Mitochondrial DNA Variant Discovery and Evaluation in Human Cardiomyopathies through Next-Generation Sequencing, 5(8), 1-9. DOI: https://doi.org/10.1371/journal. pone.0012295 